\title{
Bibliometria sobre gerenciamento de riscos: um paralelo entre anais dos principais eventos de Engenharia de Produção no Brasil
}

\author{
Marco Túlio Domingues Costa ${ }^{1}$ \\ Tábata Fernandes Pereira ${ }^{2}$ \\ Emerson José de Paiva ${ }^{3}$ \\ Daysemara Maria Cotta ${ }^{4}$
}

\begin{abstract}
RESUMO
Análise de Riscos é uma área essencial a diversas áreas profissionais. Sua importância é tal que o tema se faz sempre presente nos eventos científicos nacionais, principalmente naqueles direcionados à Engenharia de Produção. Dada sua relevância, no presente artigo apresenta-se uma análise bibliométrica sobre o tema, tomando como base as produções publicadas nos anais de dois dos principais eventos de Engenharia de Produção nacionais: o ENEGEP e o SIMPEP. Considerando o volume de artigos encontrados e extraindo desses artigos uma amostra específica de 45 trabalhos sobre o tema, entre os anos de 2010 e 2018, procedeu-se a uma avaliação acerca dos principais autores e instituições, das principais referências e dos termos chaves que caracterizam seus estudos. Como resultado, percebeu-se a existência de uma similaridade de padrões entre os anais e, principalmente, que há ainda uma carência de publicações sobre o tema "Gerenciamento de Riscos" em ambos os eventos de Engenharia de Produção.
\end{abstract}

Palavras-chave: Gerenciamento de Riscos. ENEGEP. SIMPEP. Análise Bibliométrica.

\footnotetext{
${ }^{1}$ Graduado em Engenharia de Produção pelo UNILESTE, possui Especialização em Engenharia de Segurança do Trabalho pela Faculdade Pitágoras e Mestrado em Engenharia de Produção pela Universidade Federal de Itajubá - UNIFEI, Campus Itabira. É professor e coordenador dos cursos de Engenharia da Faculdade DOCTUM, Unidade João Monlevade, MG, Brasil. E-mail: marcotuliodcosta@gmail.com.

${ }^{2}$ Graduada em Sistema de Informação pelo Centro Universitário de Itajubá - FEPI. Mestra e Doutora em Engenharia de Produção pela Universidade Federal de Itajubá - UNIFEI. É professora dos cursos de Graduação e Mestrado Profissional em Engenharia de Produção da UNIFEI, Campus Itabira, MG, Brasil. E-mail: tabatafp@unifei.edu.br.

${ }^{3}$ Graduado em Matemática pelo Centro Universitário do Sul de Minas - UNIS. Mestre e Doutor em Engenharia de Produção pela Universidade Federal de Itajubá - UNIFEI. É professor e coordenador do curso de Mestrado Profissional em Engenharia de Produção da UNIFEI, Campus Itabira, MG, Brasil. E-mail: emersonpaiva@unifei.edu.br.

${ }^{4}$ Bacharel em Engenharia de Produção pela Universidade Federal de Ouro Preto - UFOP e Mestra em Engenharia de Produção pela Universidade Federal de Minas Gerais - UFMG. É Professora do curso de Engenharia de Produção da Faculdade DOCTUM, Unidade João Monlevade, MG, Brasil. E-mail: prof.daysemara.cotta@doctum.edu.br.
} 


\title{
Bibliometry on risk management: a parallel between the production engineering brazilian main events
}

\begin{abstract}
Risk Analysis is an essential area in several professional areas. Its importance is such that the theme is always present in national scientific events, the main ones directed to Production Engineering. Due to its relevance, this article presents a bibliometric analysis on the topic, based on the productions published in the annals of two of the main national Production Engineering events: ENEGEP and SIMPEP. Considering the volume of articles found and extracting from these articles a specific sample of 45 works on the theme, between the years 2010 and 2018, an assessment was made about the main authors and institutions, the main references and the key terms that characterize your studies. As a result, it was noticed that there is a similarity patterns between annals and especially that there is still a dearth of publications on the topic "Risk Management" in both events of Production Engineering.
\end{abstract}

Keywords: Risk Management. ENEGEP. SIMPEP. Bibliometric Analysis.

Artigo recebido em: 29/04/2020

Aceito em: 04/07/2020 


\section{INTRODUÇÃO}

Independentemente do tamanho do empreendimento, do negócio ou da empresa, ou de se tratar de um projeto, de um programa ou mesmo de um portfólio, o processo de planejamento da atividade, envolve, geralmente, a avaliação de seus possíveis riscos, sejam eles físicos, financeiros ou estratégicos, de forma a nortear as decisões a serem tomadas (PMI, 2013).

A importância dada aos riscos se deve ao fato de que estes têm grande potencial de interferir e/ou comprometer o sucesso de um projeto e envolve, geralmente, dois aspectos chaves para a condução que podem afetar os objetivos traçados: o impacto que pode causar no desenrolar do projeto; e o grau de incerteza que pode trazer em relação à condução do projeto (HILLSON, 2009, apud NAPOLITANO et al., 2017).

Segundo Silva e Nascimento (2014), no cenário atual, as empresas, independentemente da área de atuação ou de seu tamanho, buscam o gerenciamento de riscos como modo de antever possíveis problemas e administrá-los para que não gerem um impacto que possa comprometer os processos ou mesmo sua imagem, sendo tal gerenciamento um fator estratégico para a organização. Assim, o gerenciamento dos riscos pode ser entendido como um mecanismo de gestão para a tomada de decisões que conduzirão, adequadamente, seja a direção dos já existentes seja a implementação de novos projetos.

Neste aspecto, conforme apontam Oliveira et al. (2015), embora o gerenciamento de projetos seja uma área valorizada, tanto no âmbito acadêmico como no profissional, há, ainda, certa escassez de estudos mais amplos ou mesmo mais personalizados dentro das diversas situações que o gerenciamento de projetos permite abordar: entre estas, o gerenciamento de riscos. Com base nisso, este estudo propõe a realização de uma análise bibliométrica sobre o gerenciamento de riscos, realizando um mapeamento de artigos que apresentam tal discussão em dois dos eventos mais importantes da engenharia de produção no país: o ENEGEP - Encontro Nacional de Engenharia de Produção e o SIMPEP Simpósio de Engenharia de Produção. Tais eventos são considerados os mais relevantes, tradicionais e com maior número de publicações dentro desta área específica de estudo. $\mathrm{O}$ mapeamento permite traçar um paralelo comparativo entre os dois eventos e apontar uma visão generalizada acerca das discussões sobre o gerenciamento de riscos.

\section{REVISÃO BIBLIOGRÁFICA}




\subsection{Projeto, gerenciamento de projetos e riscos}

As definições de projeto apresentadas são diversas e variam de um autor para outro. Adotando-se, por isso, uma generalização da definição, apoiada em PMI (2013), tem-se que o projeto é o emprego de tempo, de trabalho e de dedicação, por prazo determinado, com o intuito de se buscar soluções exclusivas, cujo término é alcançado quando os objetivos são atingidos ou quando o projeto é encerrado.

A essência desta definição extraída de PMI (2013) se faz importante, sobretudo, pela contextualização atual dos projetos desenvolvidos, em que certos aspectos antigamente negligenciados são hoje considerados importantes, tais como a delimitação de custo e de prazo, distinguindo-se de atividades contínuas em função da natureza do escopo e dos objetivos para ele definidos (KEELLING, 2002). Deve-se acrescentar, complementarmente, que o projeto possui como objetivo mínimo o atendimento das expectativas dos Stakeholders (MAIA e DI SERIO, 2016).

A compreensão conceitual do projeto é o ponto fundamental para entender a necessidade de se ter, para cada projeto, um gerenciamento dedicado. Segundo Judeng, Barbalho e Silva (2014, p. 25), o gerenciamento de projetos possui um grande destaque e uma significativa necessidade de aplicação por parte das organizações que precisam "conduzir simultaneamente diferentes projetos, dos mais simples aos mais complexos, dentro de prazos restritos estabelecidos $[. .$.$] e com qualidade e orçamentos rigorosos".$

De fato, visa a minimização de riscos com relação a custos, prazos e/ou qualidade do projeto que podem comprometer seus resultados, não atendendo ao seu planejamento inicial. Neste sentido, percebe-se que a compreensão dos riscos, em toda a amplitude do termo, deve ser considerada, para se alcançar o sucesso do projeto.

Carvalho e Rabechini Jr. (2015) explicam que o risco é intrínseco a qualquer que seja o projeto e que, por este motivo, o gerenciamento de projetos e a gestão dos riscos do projeto são atividades cuja função são quase as mesmas, cujas diferenças são bastante tênues.

Um complemento à ideia de risco, trazido por IBGC (2007), é que ele existe para qualquer pessoa ou para qualquer empresa, podendo interferir em oportunidades ou gerando perdas. Portanto, compreende-se que a dimensão dada ao gerenciamento de riscos deveria, em tese, ser, no mínimo, próxima à dada ao próprio gerenciamento de projetos. Por tal motivo, as discussões do gerenciamento de riscos são tratadas especificamente na próxima seção. 


\subsection{O gerenciamento dos riscos}

Antes de ser operacionalizado, sabendo-se que existem elementos de risco, seja qual for o projeto há a necessidade de que os responsáveis por conduzi-lo estejam certos sobre sua viabilidade, sobre quais são as potenciais ameaças que podem influenciar na sua condução, e sobre se é possível gerir estes riscos (KEELLING, 2002).

A definição de gerenciamento de riscos apresentada pelo PMI (2013) descreve que a gestão dos riscos busca elevar a perspectiva de sucesso do projeto, ao mesmo tempo em que procura mitigar condições que possam gerar impactos contraproducentes ao mesmo, empregando para isso o planejamento, a análise e o controle dos riscos aos quais o projeto está exposto.

A definição simplificada de Brandstetter \& Arantes (2015, p. 2) complementa e ajuda a entender o porquê da gestão dos riscos extrapolar a condição de aplicação exclusiva em projetos, uma vez que o autor descreve o gerenciamento de riscos como: "uma das áreas de conhecimento integrante de metodologias consolidadas de gestão", ou seja, podem ser empregadas não apenas em projetos, mas na própria gestão corporativa. Exemplo desta condição são também apresentados nos estudos de Paraskevas \& Quek (2019).

Para colocá-la em prática, a gestão de riscos utiliza uma sequência de atividades e ferramentas que possibilitam cumprir seu objetivo. Inicia-se com a identificação dos riscos, ocasião na qual se compreendem as possíveis origens dos riscos, passa pela avaliação, na qual é levantada a probabilidade e as consequências do risco, utilizando-se análises qualitativas e/ou quantitativas, planeja-se a resposta aos riscos, sobre as quais ações são tomadas para prevenir ou minimizar seus impactos, e, por fim, monitoram-se os riscos, já efetivamente na condução do projeto, fazendo-se a revisão periódica da gestão dos riscos (Clements \& Gido, 2015; PMI, 2013; Aloini et al., 2007; Keelling, 2002).

Já em relação às ferramentas, podem-se citar, no que diz respeito à identificação dos riscos: técnicas analíticas, lista de checagens, análise de históricos e opiniões especializadas; em relação à avaliação e resposta aos riscos, pode-se empregar: Estrutura Analítica de Riscos (EAR), matriz de impacto, PERT, árvore de decisão, análise de sensibilidade, matriz SWOT, entre outras (Brandstetter \& Arantes, 2015; Clements \& Gido, 2015; PMI; 2013; Keelling, 2002). Como se pode ver pelas citações, muitas das ferramentas são empregadas não apenas em projetos, mas são também amplamente utilizadas em aplicações diversas de gestão. 


\subsection{Bibliometrias correlatas}

Outras publicações já trataram de produções acadêmicas correlatas em algum nível às aqui apresentadas, empregando bibliometria para levantar trabalhos sobre gerenciamento de riscos ou referenciando congressos relevantes da área no Brasil. O Quadro 1 traz informações gerais desses estudos.

Quadro 1: Bibliometrias correlatas

\begin{tabular}{|c|c|c|c|c|}
\hline Referência & Amostra & Foco do estudo & Unidade de análise & Período de estudo \\
\hline $\begin{array}{l}\text { Barros et al. } \\
\text { (2018) }\end{array}$ & 58 artigos & $\begin{array}{c}\text { Bibliometria sobre gestão } \\
\text { de riscos baseada no } \\
\text { conhecimento }\end{array}$ & $\begin{array}{c}\text { Bases de dados } \\
\text { Web of Science, } \\
\text { Scopus e Science } \\
\text { Direct }\end{array}$ & $\begin{array}{c}\text { Entre } \\
2010 \text { e } 2018\end{array}$ \\
\hline $\begin{array}{c}\text { Ganbat et al. } \\
\text { (2018) }\end{array}$ & 527 artigos & \begin{tabular}{|} 
Bibliometria sobre uso \\
do Building Information \\
Modeling (BIM) no \\
gerenciamento de riscos
\end{tabular} & $\begin{array}{c}\text { Bases internacionais } \\
\text { diversas }\end{array}$ & $\begin{array}{c}\text { Entre } \\
2007 \text { e } 2017\end{array}$ \\
\hline $\begin{array}{l}\text { Cordeiro et } \\
\text { al. }(2016)\end{array}$ & 54 artigos & $\begin{array}{c}\text { Avaliação bibliométrica } \\
\text { de gerenciamento de } \\
\text { riscos coorporativos }\end{array}$ & $\begin{array}{l}\text { Bases de dados } \\
\text { Scopus e Spell }\end{array}$ & $\begin{array}{c}\text { Entre } \\
2005 \text { e } 2015\end{array}$ \\
\hline $\begin{array}{c}\text { Oliveira et al. } \\
\text { (2015) }\end{array}$ & 256 artigos & $\begin{array}{c}\text { Comparativo } \\
\text { bibliométrico sobre } \\
\text { gerenciamento de } \\
\text { projetos entre ENEGEP e } \\
\text { EnANPAD }\end{array}$ & $\begin{array}{l}\text { Anais do ENEGEP e } \\
\text { Anais do EnANPAD }\end{array}$ & $\begin{array}{c}\text { Entre } \\
2004 \text { e } 2014\end{array}$ \\
\hline $\begin{array}{l}\text { Peleias et al. } \\
\text { (2013) }\end{array}$ & 165 artigos & $\begin{array}{c}\text { Caracterização } \\
\text { bibliométrica } \\
\text { comparativa de gestão de } \\
\text { riscos entre EnANPAD e } \\
\text { USP }\end{array}$ & $\begin{array}{c}\text { Anais do EnANPAD } \\
\text { e } \\
\text { Anais do Congresso } \\
\text { USP }\end{array}$ & $\begin{array}{c}\text { Entre } \\
2001 \text { e } 2011\end{array}$ \\
\hline $\begin{array}{l}\text { Polo et al. } \\
\text { (2013) }\end{array}$ & 350 artigos & $\begin{array}{l}\text { Análise bibliométrica } \\
\text { sobre gerenciamento de } \\
\text { riscos publicados em } \\
\text { inglês, português e } \\
\text { espanhol }\end{array}$ & $\begin{array}{c}\text { Bases internacionais } \\
\text { diversas }\end{array}$ & $\begin{array}{c}\text { Entre } \\
2000 \text { e } 2012\end{array}$ \\
\hline $\begin{array}{l}\text { Bueno } \\
(2011)\end{array}$ & 66 artigos & $\begin{array}{l}\text { Análise de artigos } \\
\text { alinhados ao PMBOK } \\
\text { com ênfase em RH, } \\
\text { Qualidade e Riscos }\end{array}$ & Anais do ENEGEP & $\begin{array}{c}\text { Entre } \\
2008 \text { e } 2010\end{array}$ \\
\hline
\end{tabular}

Fonte: Autoria própria. 
Todos os estudos ora apresentados possuem algum grau de similaridade com a proposta deste trabalho, sobretudo com o enfoque no gerenciamento de riscos. Embora o trabalho de Oliveira et al. (2015) tenha focado no gerenciamento de projetos como um todo, e não especificamente no gerenciamento de riscos, este foi considerado por se compreender que há relevância significativa acerca do comparativo entre dois congressos de referência no Brasil e suas posteriores análises para o desenvolvimento do presente trabalho.

Os trabalhos de Calixto et al. (2012) e Oliveira et al. (2015) apresentam outras bibliometrias realizadas especificamente sobre o gerenciamento de projetos ou correlações diretas com este tema. Entretanto, quando avaliado o gerenciamento de riscos, percebe-se que há um campo maior a explorar em relação às análises bibliométricas, uma vez que há poucos estudos desse formato, sobretudo se se consideram os eventos nacionais da área.

\section{METODOLOGIA}

Diante do contexto apresentado neste estudo, em que se aborda o gerenciamento de riscos de projetos, este artigo realiza um mapeamento do tema considerando os dois principais congressos brasileiros de Engenharia de Produção.

Para cumprir este objetivo, foi realizada uma pesquisa bibliográfica juntamente com uma análise bibliométrica sobre essa temática. Segundo Oliveira et al. (2015, p. 20), a análise bibliométrica: “consiste em uma técnica para medição dos índices de produção e divulgação do conhecimento científico, o qual permite obter informações em uma área acadêmica, e serve, entre outras funções, para demonstrar características relacionadas à produção científica desta área".

Para que a análise bibliométrica pudesse ser realizada, foram selecionados dois principais e mais antigos congressos nacionais de Engenharia de Produção. Sendo eles o Encontro Nacional de Engenharia de Produção (ENEGEP), que possui 38 eventos até 2018, e o Simpósio de Engenharia de Produção (SIMPEP), com 25 ocorrências até este ano.

A fim de se obterem os dados para o trabalho, foi estabelecido o período de dez anos para a análise (2010 a 2018). Os anais desses congressos foram consultados e as pesquisas foram realizadas nestas bases de dados, podendo-se obter os dados sobre o gerenciamento de riscos.

Dessa forma, para se conduzir a análise bibliométrica, foi elaborado um passo a passo que foi baseado na proposta de Maia \& Siero (2012). Esse passo a passo (Figura 1) teve 
como objetivo facilitar o andamento da análise bibliométrica conduzida, neste caso, em anais de congressos.

Conforme apresentado na Figura 1, foram selecionados os dois principais congressos em Engenharia de Produção: ENEGEP e SIMPEP. Na parte superior da Figura 1, iniciamse as etapas da pesquisa nos anais do ENEGEP. A primeira etapa consiste em definir as palavras-chaves e, em seguida, analisa-se se é necessária a aplicação de filtros, tais como área de conhecimento ou tipo de publicação. Após essa avaliação, encaminha-se para a etapa de análise descritiva desses artigos. O mesmo procedimento é realizado para o congresso SIMPEP, seguindo as mesmas etapas. Ao final da análise descritiva, os dados de ambos congressos são combinados e, por fim, as comparações e conclusões são realizadas.

Figura 1: Roteiro para Análises Bibliométrica em congresso

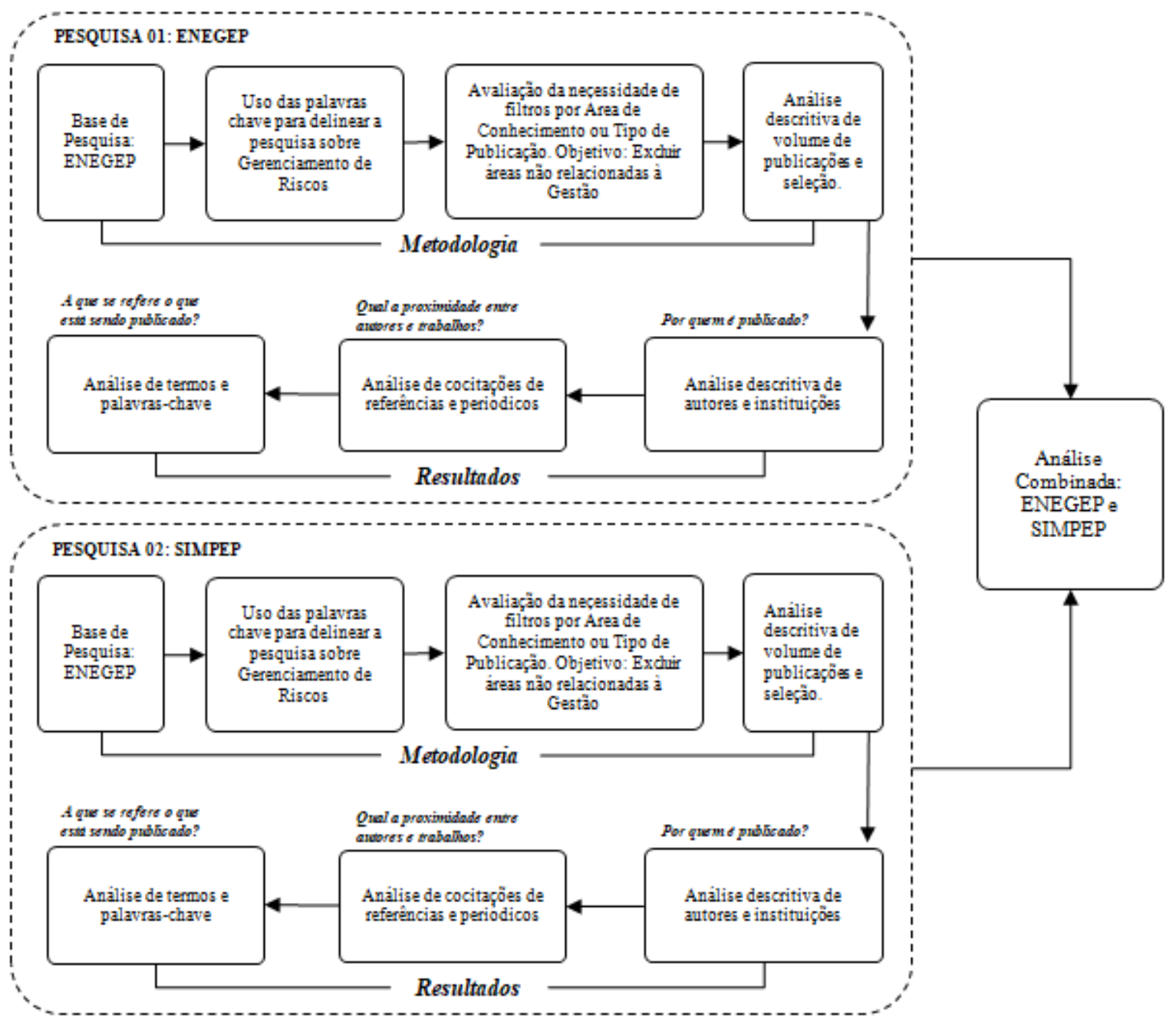

Fonte: Adaptado de Maia \& Siero (2016).

O passo a passo da Figura 1 foi seguido e, como resultado da análise, pode-se obter um total de 17.126 artigos sobre gerenciamento de riscos que foram publicados entre 2010 
e 2018 nos dois anais dos congressos. O volume de publicações ano a ano é apresentado na Figura 2.

Assim, a amostra foi obtida de um total de 10.203 trabalhos publicados no ENEGEP e outros 6.923 trabalhos publicados nos anais do SIMPEP. O volume amostrado é significativamente menor, uma vez que, como já colocado, se restringiu apenas às publicações relacionadas ao gerenciamento de riscos. Vale ressaltar que foram excluídos os trabalhos ligados à gestão de riscos ocupacionais, por compreender-se que as ferramentas e técnicas aplicadas são distintas e o objetivo dessa área tem propósito diferente daquela que se pretende levantar neste estudo. Assim, os trabalhos ligados às áreas de segurança e saúde do trabalho foram desconsiderados nesta análise bibliométrica.

Figura 2: Total de publicações anuais por anais.

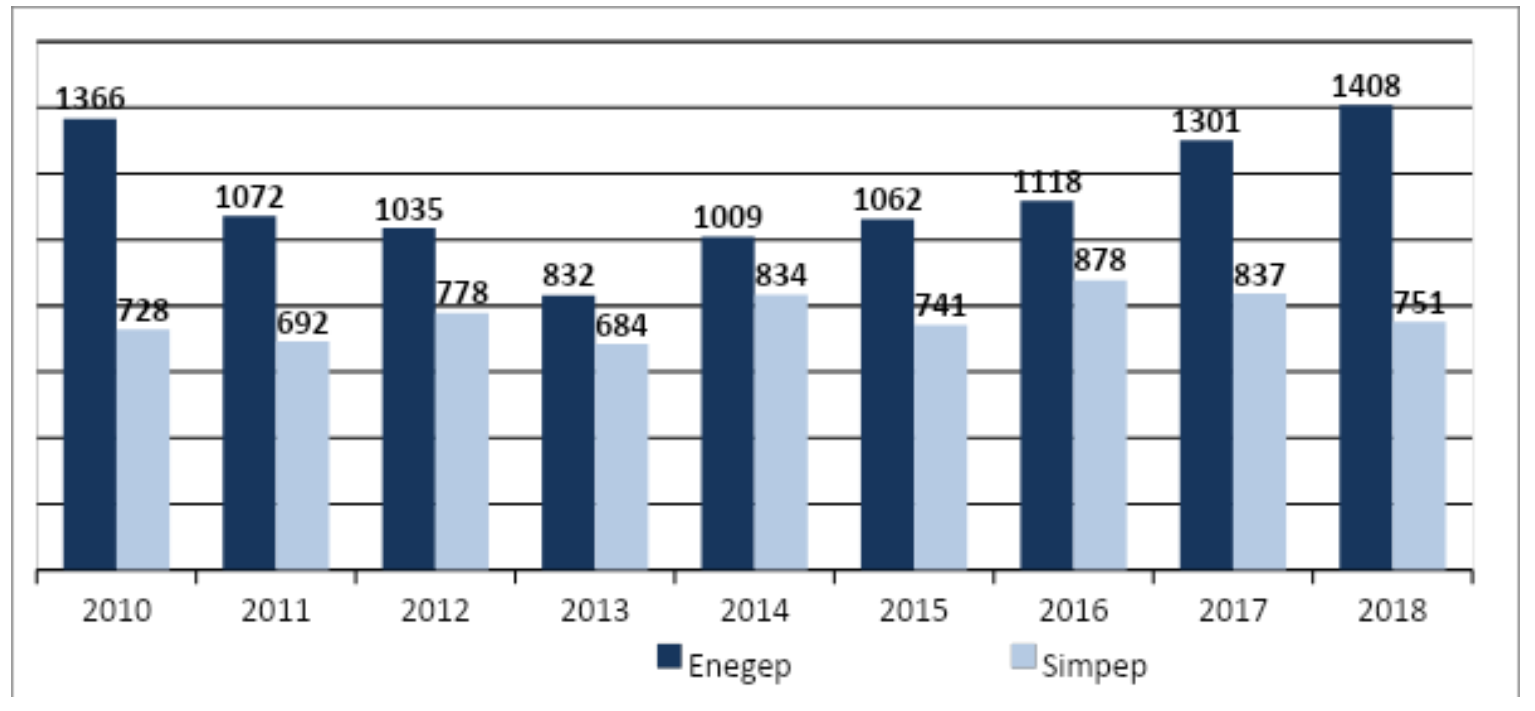

Fonte: Autoria própria.

Dessa forma, após a análise dos artigos e a exclusão daqueles que não estavam relacionados ao tema desta pesquisa, pode-se chegar a uma amostra de 45 trabalhos, distribuídos ao longo dos 10 anos da pesquisa, conforme é apresentado na Figura 3 (a seguir). 
Figura 3: Total de publicações anuais por anais

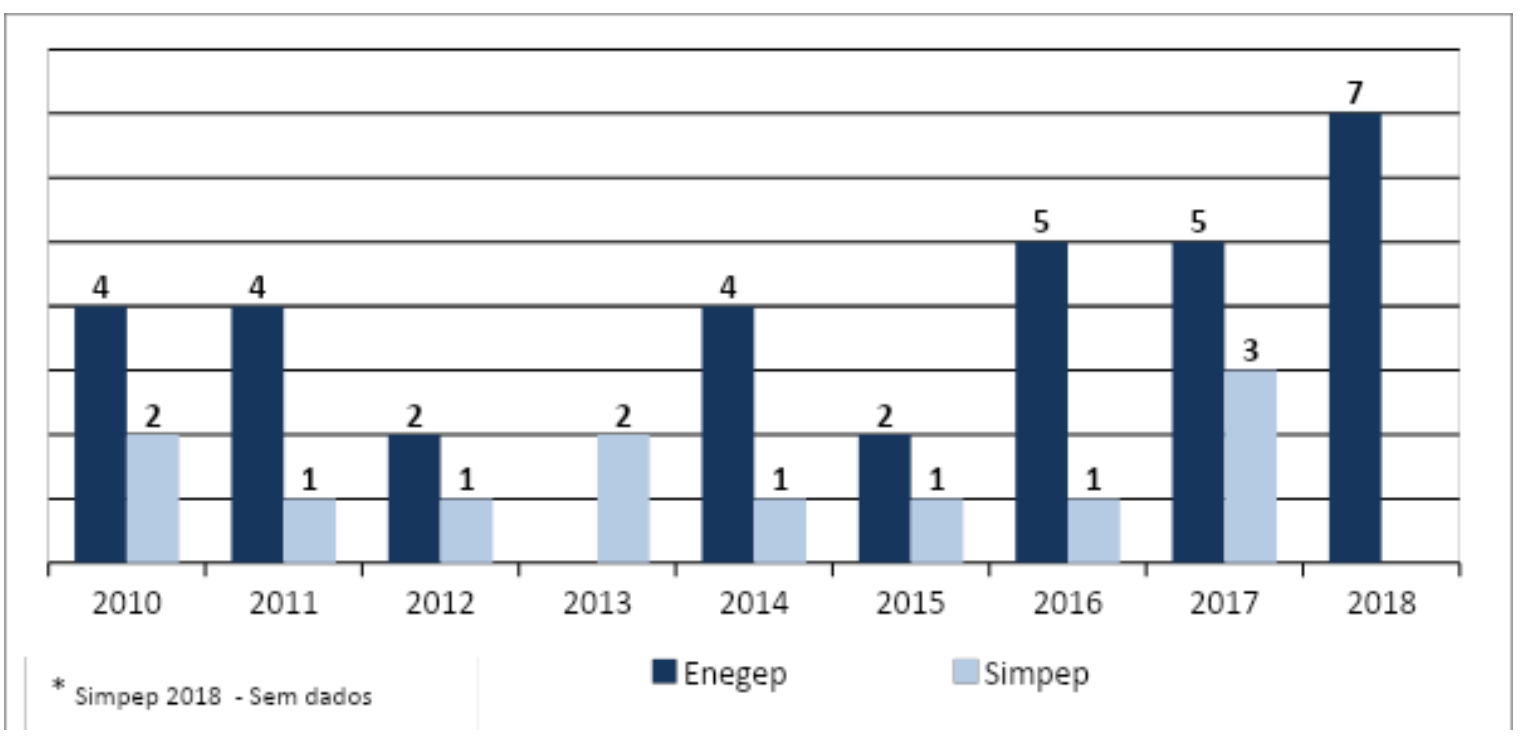

Fonte: Autoria própria.

Conforme observado na Figura 3, há uma evolução tímida no número de publicações ao longo dos anos estudados apenas no ENEGEP. Pode-se perceber também que o volume de publicações no ENEGEP sobre o tema é, em quase todos os anos, mais que o dobro do número de publicações no SIMPEP, estando aquele sempre à frente deste em número de publicações sobre o tema, com a exceção do ano de 2013, no qual não houve publicações relacionadas ao gerenciamento de riscos no ENEGEP. O conjunto dessas publicações forma a amostra adotada no estudo que será considerada na próxima seção.

\section{ANÁLISE E RESULTADOS}

Baseado na amostragem descrita na seção anterior, compreende-se que a unidade de análise considerando os anais do ENEGEP são 33 artigos, ao passo que a unidade de análise dos anais do SIMPEP (sem considerar o ano de 2018 que não havia sido divulgado até a realização deste estudo) são 12 trabalhos, totalizando 45 publicações no período estudado.

\subsection{Análise de autores e instituições}

Analisando os autores de cada artigo, incluindo os coautores apresentados nas publicações, percebe-se que o número de pessoas envolvidas nos artigos publicados, em ambos os congressos, é aleatório, havendo pouca repetição de autores. Ao todo, foi identificado um total de 131 autores e coautores dos trabalhos publicados nos anais dos dois 
eventos, sendo 40 no SIMPEP e 91 no ENEGEP. Para a referida área de pesquisa, não foi identificado nenhum autor que tenha publicado sobre o gerenciamento de riscos nos dois eventos estudados. A Tabela 1 apresenta, para cada evento, os autores com mais de uma publicação sobre o tema em cada um dos anais.

Tabela 1: Autores com maior número de publicações

\begin{tabular}{|c|c|c|c|}
\hline \multicolumn{2}{|c|}{ ENEGEP } & \multicolumn{2}{c|}{ SIMPEP } \\
\hline Autor & Publicações & Autor & Publicações \\
\hline $\begin{array}{c}\text { Carlos Eduardo Sanches da } \\
\text { Silva }\end{array}$ & 5 & Eduardo Vieira do Prado & 2 \\
\hline Marcelo Hazin Alencar & 4 & Antônio Carlos Giulianni & 2 \\
\hline Sandra Miranda Neves & 4 & & \\
\hline $\begin{array}{c}\text { Lucas Lobato Pio Dos Santos } \\
\text { Hora }\end{array}$ & 2 & & \\
\hline Luiz Priori Junior & 2 & & \\
\hline Tábata Fernandes Pereira & 2 & & \\
\hline Aneirson Francisco da Silva & 2 & & \\
\hline
\end{tabular}

Fonte: Autoria própria.

Conforme observa-se pela Tabela 1 , a repetição de autoria é muito mais comum nos anais do ENEGEP. Um fato que se deve destacar ainda é que, considerando os autores apresentados na referida tabela, com a exceção de Aneirson Francisco da Silva, todos os demais autores foram identificados exatamente em duas publicações, tendo feito estas na mesma edição de cada evento.

Quando se avaliam as publicações em função das instituições de origem, pode-se identificar que os trabalhos são de pesquisadores de 37 diferentes instituições, sendo 15 instituições públicas e as demais, privadas. Vale destacar que a amplitude do número de instituições é ligeiramente menor no SIMPEP, com publicações oriundas de 18 instituições, ao passo que no ENEGEP identificam-se 23 instituições de origem, sendo que cinco delas são comuns aos dois anais pesquisados. Dois trabalhos do ENEGEP não tiveram as instituições de origem identificadas. A distribuição do número de artigos entre as instituições que mais publicaram é apresentada na Tabela 2 (a seguir). 
Tabela 2: Instituições com maior número de publicações

\begin{tabular}{|c|c|c|c|c|c|}
\hline \multicolumn{2}{|c|}{ ENEGEP } & \multicolumn{2}{c|}{ SIMPEP } & \multicolumn{2}{c|}{ Total } \\
\hline Instituição & Publicações & Instituição & Publicações & Instituição & Publicações \\
\hline UFPE & 8 & UFRJ & 2 & UFPE & 8 \\
\hline UNIFEI & 7 & UNIMEP & 2 & UNIFEI & 7 \\
\hline FEI & 2 & USP & 2 & UNINOVE & 3 \\
\hline UNINOVE & 2 & & & USP & 2 \\
\hline & & & & SENAI-RJ & 2 \\
\hline & & & & UFRJ & 2 \\
\hline & & & & UFRN & 2 \\
\hline & & & & UFSM & 2 \\
\hline & & & & UNIMEP & 2 \\
\hline & & & & Outras & 13 \\
\hline
\end{tabular}

Fonte: Autoria própria.

Como se pode observar na Tabela 2, das 37 instituições que tiveram artigos publicados nestes eventos, apenas 10 publicaram sobre gerenciamento de riscos em mais de uma ocasião. Nesse quesito, duas instituições se sobressaem: a UFPE e a UNIFEI, ambas com um número de publicações consideravelmente maior que o das demais.

Quando considerados os estados de origem das instituições, percebe-se também que não há grande diversidade, sendo que, das 27 unidades federativas, apenas instituições de 11 estados estão representadas, havendo predominância das publicações de estados das regiões Sudeste, Sul e Nordeste. Não foi identificado nenhum trabalho de instituições da região Norte. A Tabela 3 apresenta o número de publicações por estado.

Tabela 3: Publicações por estados

\begin{tabular}{|c|c|c|c|c|c|}
\hline \multicolumn{2}{|c|}{ ENEGEP } & \multicolumn{2}{c|}{ SIMPEP } & \multicolumn{2}{c|}{ Total } \\
\hline Estado & Publicações & Estado & Publicações & Estado & Publicações \\
\hline PE & 8 & SP & 5 & SP & 10 \\
\hline MG & 7 & RJ & 3 & MG & 8 \\
\hline SP & 5 & BA & 1 & PE & 8 \\
\hline GO & 4 & GO & 1 & RJ & 4 \\
\hline
\end{tabular}

Continua... 
... Conclusão

\begin{tabular}{|c|c|c|c|c|c|}
\hline \multicolumn{2}{|c|}{ ENEGEP } & \multicolumn{2}{c|}{ SIMPEP } & \multicolumn{2}{c|}{ Total } \\
\hline Estado & Publicações & Estado & Publicações & Estado & Publicações \\
\hline SC & 2 & MG & 1 & GO & 2 \\
\hline CE & 1 & PR & 1 & PR & 2 \\
\hline PR & 1 & RN & 1 & RN & 2 \\
\hline RJ & 1 & RS & 1 & RS & 2 \\
\hline RN & 1 & & & BA & 1 \\
\hline RS & 1 & & & CE & 1 \\
\hline & & & & SC & 1 \\
\hline
\end{tabular}

Fonte: Autoria própria.

Como se observa na Tabela 3, há predominância de publicações de instituições da região Sudeste sobre gerenciamento de riscos nos principais eventos de engenharia de produção, totalizando-se 22 publicações oriundas dos três estados: São Paulo, Minas Gerais e Rio de Janeiro (não há publicações do Espírito Santo), o que representa quase metade das publicações da área, 46,8\% do total. O Nordeste vem na sequência, com 25,5\% das publicações. Por fim, com 10,6\%, o Sul se apresenta com um número de publicações razoável.

Vale destacar que, enquanto há uma dispersão entre várias instituições paulistas, nos outros dois estados com mais publicações, Minas Gerais e Pernambuco, elas vêm praticamente das mesmas instituições, UNIFEI e UFPE, respectivamente. Os dois autores com maior repetição são também dessas respectivas instituições, o que pode demonstrar um padrão de estudo desses pesquisadores e instituições.

\subsection{Análise de citações e co-citações}

Em relação às referências empregadas pelos autores e citadas nos artigos publicados em ambos os congressos, percebem-se alguns pontos divergentes; no entanto, existem também grandes similaridades. O primeiro aspecto avaliado diz respeito ao volume de materiais consultados. No ENEGEP, por exemplo, somando-se as referências de todos os artigos, obtém-se um total de 655 publicações, sendo 565 materiais distintos. Nas demais 90 referências $(13,7 \%)$, há repetição de trabalhos. Na média, cada artigo publicado referência cerca de 20 trabalhos. No SIMPEP o volume de publicações é similar. 
Em relação ao tipo de material pesquisado, há predominância em ambos congressos. A Figura 4 apresenta os resultados encontrados para as referências utilizadas por evento.

Figura 4: Tipos de referências utilizadas por evento

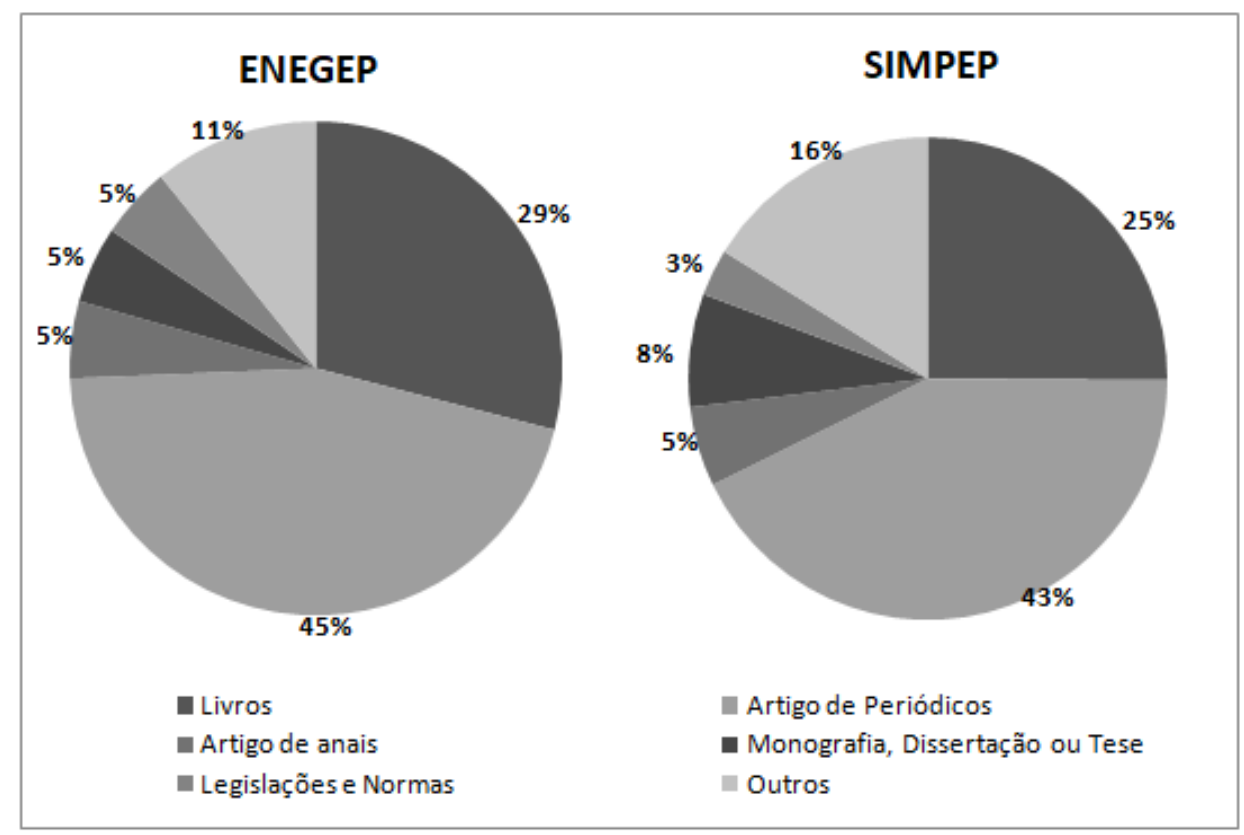

Fonte: Autoria própria.

Como se pode perceber, tanto para o ENEGEP como para o SIMPEP quase metade das referências são de trabalhos publicados em periódicos. Em seguida, o uso de livros como fonte de consulta vem em segundo lugar também para ambos os eventos, sendo quase um terço das consultas no ENEGEP e exatamente um quarto no SIMPEP. As demais fontes aparecem em uma proporção menor. Vale destacar o aspecto de citações e referências de artigos publicados em anais de eventos serem pouco expressivos, uma vez que aqui se estuda justamente esse tipo de publicação.

Neste ponto, ressalta-se que em nenhum artigo publicado no SIMPEP há citações de outros trabalhos publicados neste mesmo evento. No ENEGEP existe a utilização de artigos publicados no próprio evento, mas em baixo número, sendo apenas seis ocorrências (menos de $1 \%$ do total de materiais consultados).

Como já apresentando, houve um número muito pequeno de repetição das referências utilizadas, mas dentre aquelas que tiveram citações em mais de um artigo, duas se destacam: o PMBOK e a NBR ISSO 31000. Embora nenhuma das duas referências esteja presente em todos os trabalhos, percebe-se que estão entre as principais fontes de consulta para esta área de estudo, sendo o primeiro citado em pouco mais da metade dos trabalhos avaliados, 
enquanto o segundo foi percebido em quase um terço das publicações. A listagem das principais referências está apresentada na Tabela 4.

Tabela 4: Bibliografias com maior número de citações

\begin{tabular}{|c|c|c|c|}
\hline Referência & ENEGEP & SIMPEP & Total \\
\hline $\begin{array}{l}\text { PMI. PMBOK® Guide: A Guide to the Project Management } \\
\text { Body of Knowledge (PMBOK® Guide). } 3^{\mathrm{a}} / 4^{\mathrm{a}} / 5^{\mathrm{a}} \text { Ed. }\end{array}$ & 17 & 6 & 23 \\
\hline $\begin{array}{l}\text { ABNT NBR ISO } 31.000 \text { - Gestão de riscos - Princípios e } \\
\text { diretrizes. Rio de Janeiro: ABNT, } 2009 .\end{array}$ & 7 & 4 & 11 \\
\hline $\begin{array}{l}\text { KERZNER, H. Gestão de Projetos: As Melhores Práticas. 2.ed. } \\
\text { Porto Alegre: Bookman, p.328-342, } 2006 .\end{array}$ & 5 & 1 & 6 \\
\hline $\begin{array}{l}\text { YIN, Robert. Estudo de Caso: Planejamento e Métodos. } 5 \text { ed. } \\
\text { Porto Alegre: Bookman, } 2015 .\end{array}$ & 4 & 1 & 5 \\
\hline $\begin{array}{l}\text { CARVALHO, M. M.; RABECHINI JUNIOR, R. Fundamentos } \\
\text { em Gestão de Projetos: Construindo Competências para } \\
\text { Gerenciar Projetos. 3. ed. São Paulo: Atlas, } 2011\end{array}$ & 3 & 2 & 5 \\
\hline $\begin{array}{l}\text { COSO-Committee of Sponsoring Organizations of the Treadway } \\
\text { Commission. Gerenciamento de Riscos Corporativos - Estrutura } \\
\text { Integrada, } 2007 \text {. }\end{array}$ & 3 & 2 & 5 \\
\hline $\begin{array}{l}\text { HILLSON, D. , Extending the Risk Process to Manage } \\
\text { Opportunities. Proceedings of the Fourth European Project } \\
\text { Management Conference, PMI Europe 2001, 6-7 June 2001, } \\
\text { London UK. }\end{array}$ & 3 & 2 & 5 \\
\hline $\begin{array}{l}\text { RAZ, T.; SHENHAR, A. J. \& DVIR, D. Risk management, } \\
\text { project success, and technological uncertainty. R\&D } \\
\text { Management. Vol. 32, n. 2, p. 101-109, } 2002\end{array}$ & 3 & 1 & 4 \\
\hline $\begin{array}{l}\text { ALMEIDA A.T., et al. Multicriteria and Multi-objective Models } \\
\text { for Risk, Reliability and Maintenance Decision Analysis. In: } \\
\text { Operations Research \& Management Science. Vol. 231. 387p, } \\
2016\end{array}$ & 4 & 0 & 4 \\
\hline $\begin{array}{l}\text { GIL, A. C. Como elaborar projetos de pesquisa. 5. ed. São Paulo: } \\
\text { Atlas, } 2010 .\end{array}$ & 4 & 0 & 4 \\
\hline
\end{tabular}

Fonte: Autoria própria.

Além das duas referências mais citadas, nota-se, por meio da Tabela 4, outros dois aspectos: o primeiro é a predominância de livros, o que mostra, de uma forma generalizada, o porquê de não ter sido dada atenção a artigos de grande relevância na literatura. O segundo aspecto é que, nesta mesma lista, estão entre os mais citados dois livros de metodologia científica, que não têm relação direta com o tema estudado. 
Cabe destacar que outros autores tiveram número de citações similares, R. L. Rovai com cinco e M. Christopher com quatro citações. Em todos os trabalhos analisados há apenas um artigo em que se constata a citação de um autor por outro que também publicou sobre gerenciamento de riscos no ENEGEP. Não houve citações entre autores no SIMPEP, tampouco referências cruzadas entre os dois anais.

\subsection{Análise dos termos e palavras-chaves}

A análise dos termos e das palavras-chaves foi dividida em dois blocos principais: o primeiro diz respeito especificamente às palavras-chaves apresentadas em cada trabalho publicado, enquanto o segundo refere-se à inclinação da publicação baseada no título do projeto, independentemente de os termos terem sido ou não empregados como palavraschaves.

Em relação ao conjunto de palavras-chaves identificadas, compreende-se que não houve nenhuma repetição efetiva ao ponto de identificar alguma tendência de estudos. A média geral para ambos os anais é de três palavras-chaves por artigo. Aquelas que foram citadas mais de uma vez estão apresentadas na Tabela 5.

Tabela 5: Relação de palavras-chaves mais citadas

\begin{tabular}{|c|c|c|c|c|}
\hline Palavra-chave & Sinônimo aceito & ENEGEP & SIMPEP & Total \\
\hline Gerenciamento de Riscos & Gestão de riscos & 20 & 11 & 31 \\
\hline Gerenciamento de Projetos & Gestão de projetos & 9 & 1 & 10 \\
\hline FMEA & & 3 & 2 & 5 \\
\hline Simulação de Monte Carlo & Monte Carlo & 1 & 2 & 3 \\
\hline $\begin{array}{c}\text { Ferramenta de Gerenciamento de } \\
\text { riscos }\end{array}$ & & 1 & 1 & 2 \\
\hline Análise de riscos & & 1 & 1 & 2 \\
\hline Governança corporativa & & 1 & 1 & 2 \\
\hline Análise bibliométrica & & 2 & 0 & 2 \\
\hline Análise de risco & & 2 & 0 & 2 \\
\hline Construção Civil & Plantas industriais & 2 & 0 & 2 \\
\hline Empreendimentos industriais & & & & 1 \\
\hline
\end{tabular}

Continua... 
... Conclusão

\begin{tabular}{|c|c|c|c|c|}
\hline Palavra-chave & Sinônimo aceito & ENEGEP & SIMPEP & Total \\
\hline Desastres naturais & & 2 & 0 & 2 \\
\hline Riscos & & 2 & 0 & 2 \\
\hline
\end{tabular}

Fonte: Autoria própria.

Por meio da Tabela 5 nota-se uma tendência de repetição dos dois primeiros termos, o que já era esperado tendo em vista a própria escolha dos artigos. Considera-se, assim, que tais termos não são suficientes para explicar ou levantar qualquer parâmetro de publicação. Quando considerada as demais palavras-chaves, entretanto, percebe-se que não há nenhuma tendência efetiva que implique um padrão identificável, uma vez que o número de repetições é pequeno e muitos dos termos indicados estão intrinsecamente ligados à prática de gerenciamento de riscos, como é o caso, por exemplo, do terceiro termo mais utilizado, FMEA, que é uma ferramenta dedicada a identificar possibilidades de falhas em projetos.

Uma visão similar pode ser observada com relação à avaliação da inclinação dos estudos. De uma forma geral, o que se pôde perceber é a aplicação do gerenciamento de riscos de forma pulverizada, não havendo nenhuma área com um destaque efetivo. As informações acerca desta inclinação são apresentadas na Tabela 6.

Tabela 6: Inclinação dos estudos publicados

\begin{tabular}{|l|c|c|c|}
\hline \multicolumn{1}{|c|}{ Inclinação do estudo } & ENEGEP & SIMPEP & Total \\
\hline Cadeia de Suprimentos & 2 & 1 & 3 \\
\hline Desenvolvimento de Software & 3 & 0 & 3 \\
\hline Indústria Automotiva & 1 & 1 & 2 \\
\hline Processos Industriais & 2 & 0 & 2 \\
\hline Governança Corporativa & 2 & 0 & 2 \\
\hline Suporte às Decisões & 2 & 0 & 2 \\
\hline Gestão do Conhecimento & 2 & 0 & 2 \\
\hline
\end{tabular}

Fonte: Autoria própria.

Como se pode observar na Tabela 6, o número de temas que se repetiram nos estudos é pequeno, havendo recorrências, seja no mesmo evento ou entre os dois anais, apenas em sete áreas. Não houve nenhuma repetição de área nos artigos publicados nos anais do 
SIMPEP. Ainda assim, deve-se destacar que estas repetições representam pouco mais de um terço do total de trabalhos avaliados e que é inapropriado discernir sobre qualquer tipo de prevalência.

\section{CONSIDERAÇÕES FINAIS}

As publicações em anais de congressos e simpósios são meios interessantes de divulgação de pesquisas, troca de ideias, interação entre pesquisadores, fomento e transmissão de conhecimentos científicos entre os envolvidos com uma determinada área, como é o caso proposto dos dois eventos de engenharia de produção apontados neste estudo bibliométrico. Desses anais, muitas informações podem ser levantadas e empregadas por pessoas com interesse em determinada linha de estudo, constituindo, assim, uma base sólida de referência de publicações para estudantes, especificamente para aqueles ligados à engenharia de produção.

Compreende-se que há a necessidade de que se tenham boas publicações, respaldadas por referências clássicas de livros, de publicações relevantes acerca do tema em estudo, além daquelas publicações recentes, que ditam os rumos do tema na atualidade.

$\mathrm{Na}$ presente análise bibliométrica, buscou-se tratar especificamente de um tema relevante e útil para a aplicação no dia a dia das empresas e, também, para novos projetos. Tratou-se, aqui, das publicações acerca do gerenciamento de riscos que, embora muitas vezes seja tratado como um subtema do gerenciamento de projetos, possui uma importância própria, tal qual o próprio gerenciamento de projetos.

É sabido, todavia, que existem eventos e periódicos dedicados exclusivamente à apresentação de práticas e inovações nas áreas de gerenciamento de projetos. Mas compreendendo, primeiramente, que o gerenciamento de riscos extrapola essa aplicação e também deve ser trabalhado em outras condições empresariais e, em segundo lugar, que eventos gerais de determinados cursos apresentam, também, transversalidades em mais de uma área que lhes compõem, pode-se afirmar que os principais eventos de um curso devem trazer aspectos relevantes e suficientes que sirvam de base para novos estudos.

Nesse sentido, foi possível perceber dois pontos principais: o primeiro é que há uma similaridade entre os padrões de proporcionalidade e características das publicações entre os dois anais, muito embora as instituições e os autores envolvidos sejam distintos entre ambos; o segundo é que há, ainda, pouca exploração do tema de gerenciamento de riscos em ambos 
os anais estudados, não sendo possível distinguir nenhum foco de pesquisa mais efetivo. Além disso, as informações obtidas sobre as publicações correlatas ao gerenciamento de riscos chegam a ser insuficientes para as pretensões deste estudo. Observou-se, por exemplo, a falta de pesquisa e de referências a trabalhos publicados em ambos os anais nos próprios artigos submetidos àqueles eventos.

Considera-se, portanto, que o estudo sobre gerenciamento de riscos e sua publicação nos referidos anais possuem um campo amplo de exploração, apresentando-se, ainda que timidamente, em fase de construção em ambos os eventos.

Como trabalhos futuros, sugere-se a pesquisa bibliográfica do tema em periódicos de circulação nacional, um estudo comparativo entre o volume de estudos sobre o tema em periódicos e anais, além de uma pesquisa mais aprofundada sobre o tema no âmbito de periódicos estrangeiros.

\section{REFERÊNCIAS}

ALOINI, D.; DUlmin, R.; MINNINO, V. Risk Management in ERP Project introduction: Review of literature. Information and Management, 44, p. 547-567, 2007.

BRAnDStetTer, M. C. G. O.; ARAnTES, G. M. Proposições de aplicação do gerenciamento de riscos em sistemas da construção. Anais do Encontro Nacional de Engenharia de Produção, ENEGEP. Fortaleza, CE, Brasil, 35, 2015.

BARROS, I. J. R. C.; NEVES, S. M.; OLIVEIRA, C. H.; PEREIRA, T. F.; SANTOS, P. H. Gestão de Riscos baseada no conhecimento: uma análise bibliométrica com ênfase em projetos de software. Anais do Encontro Nacional de Engenharia de Produção, ENEGEP. Maceió, AL, Brasil, 38, 2018

BUENO, J. M. Produção científica em Gestão de Projetos: um balanço crítico sobre pesquisas na área. Anais do Encontro Nacional de Engenharia de Produção, ENEGEP. Belo Horizonte, MG, Brasil, 31, 2011.

CALIXTO, G. M.; FURLAN, P. K.; CARVALHO, M. M. Estudo das tendências na análise de competências na gestão de projetos através de técnicas de bibliometria. Revista de Gestão e Projetos - GeP, 3(1), p. 181-196, 2012.

CARVAlHO, M. M.; RABECHINI JR., R. Fundamentos em Gestão de Projetos: Construindo Competências para Gerenciar Projetos. $4^{\mathrm{a}}$ ed. São Paulo: Editora Atlas, 2015.

CLEMENTS, J. P.; GIDO, J. Successful Project Management. 6 th Ed. New York: Cengage Learning, 2015.

CORDEIRO, J. F.; RECH, I. J.; CRUZ, A. F. Análise bibliométrica da produção científica 
sobre risco corporativo no período de 2005 a 2015. Anais do Congresso Brasileiro de Custos. Porto de Galinhas, PE, Brasil, 23, 2016.

GANBAT, T.; CHONG, H.; LIAO, P.; WU, Y. A bibliometric revew on risk management and building information for international construction. Hindawi Advanceds in Civil Engineering, p. 1-13, 2018.

IBGC - Instituto Brasileiro de Governança Corporativa. Guia de Orientação para Gerenciamento de Riscos Corporativos, 2007.

JUDENG, D.; BARBALHO, S. C. M.; SILVA, S. L. Gestão de projetos: teoria, prática e tendências. Rio de Janeiro: Elsevier, 2014.

KEELLING, R. Gestão de Projetos: Uma abordagem Global. São Paulo: Saraiva, 2002.

MAIA, J. L.; DI SERIO, L. C. Uma análise bibliométrica multifontes sobre a geração de valor ao acionista e a gestão de projetos, programas e portfólio. Revista de Gestão e Projetos - GeP, 8 (2), p. 87-108, 2016.

NAPOLITANO, D. M. R.; FERREIRA, R. P.; SASSI, R. J. Sistema de inferência Fuzzy na elaboração de matrizes de risco para a análise qualitativa em projetos. Anais do Encontro Nacional de Engenharia de Produção, ENEGEP. Joinvile, SC, Brasil, 37, 2017.

OLIVEIRA, R. R.; BOLDORINI, P. S. A. T.; MARTINS, H. C.; DIAS, A. T. Gerenciamento de projetos: comparativo bibliométrico dos anais de congressos brasileiros na área de administração e engenharia de produção. Revista de Gestão $e$ Projetos - GeP, 7 (1), p. 15-31.

PARASKEVAS, A.; QUEK, M. When Castro seized the Hilton: Risk and crisis management lessons from the past. Tourism Management, 70, p. 419-429, 2019.

PELEIAS, I. R.; CAETANO, G.; PARISI, C.; PEREIRA, A. C. Produção científica sobre controle interno e gestão de riscos no EnANPAD e congresso USP: análise bibliométrica no período 2001-2011. Revista Universo Contábil, 9 (4), p. 29-49, 2013.

PMI - Project Management Institute. Pmbok, Guide. Um guia do conhecimento em gerenciamento de projetos, 2013.

POLO, E.; PEIXE, M.; GALEGALE, G. Estudo Bibliométrico da Produção Científica de Gerenciamento de Riscos entre os Anos de 2000 e 2012. CONF-IRM Proceedings, 31, 2013.

SILVA, C. E. S.; NASCIMENTO, K. M. Análise da incorporação da incerteza no gerenciamento de riscos para o atendimento do prazo em projetos de pesquisa \& desenvolvimento. Anais do Encontro Nacional de Engenharia de Produção, ENEGEP, Curitiba, PR, Brasil, 34, 2014. 